\title{
Percepciones del alumnado universitario sobre el aprendizaje-servicio como herramienta para su desarrollo personal y profesional
}

\author{
Virginia Martínez Lozano \\ Rosa Ma Rodríguez Izquierdo \\ María Marco Macarro \\ Beatriz Macías Gómez-Estern \\ Universidad Pablo de Olavide, España
}

\section{Resumen}

El artículo presenta una experiencia de innovación docente desarrollada con metodología de aprendizaje-servicio en el primer curso del Grado de Educación Social de una universidad pública española. La experiencia implica a dos asignaturas llevando a cabo un servicio comunitario en un C.E.I.P. constituido como Comunidad de Aprendizaje en una zona con necesidades de transformación social, con población mayoritaria de etnia gitana. Mostramos los resultados sobre las percepciones de los estudiantes sobre los aprendizajes adquiridos durante la experiencia. Los resultados se han organizado clasificando los aprendizajes percibidos en curricularesprofesionales; sobre la realidad social; y sobre sí mismos. Se analizaron también las valoraciones sobre la metodología empleada. Los datos muestran que el estudiantado destaca especialmente los aprendizajes personales. Concluimos que la metodología empleada es una buena herramienta docente generadora de motivación por el aprendizaje y de futuros profesionales comprometidos.

\section{Palabras clave}

Aprendizaje-servicio, aprendizaje auténtico, identidad profesional, identidad personal, educación superior. 


\title{
University students' perceptions on service-learning as a tool for personal and professional development
}

\begin{abstract}
This article presents a teaching innovation experience developed in the first year of a Social Education Degree at a public university in Spain, using a service-learning methodology. The experience involves two disciplines from this degree program working collaboratively and the results show students' perceptions on the personal and professional skills they acquired. The proposal is based not only on the notion of SL as a tool for the construction of committed and active citizenship, but from the consideration of SL as a methodology able to produce authentic learning, which involves transformations that go much further than cognitive changes and affect students' own personal construction. We have used two sets of students' narratives: a questionnaire about their learning perceptions and another on their reflections throughout the experience. The data shows that students more emphatically refer to personal learning, followed by curricular-professional learning and finally learning about the reality. We also found that evaluation of the experience was mainly positive, pointing especially to the idea of learning by doing. We conclude that the methodology used generates in the students personal and social conceptions that go beyond what is achieved with traditional methodologies, showing that SL can be considered as an optimal teaching methodology to generate motivation for learning and commitment in future professionals.
\end{abstract}

\section{Keywords}

Service-learning, authentic learning, professional identity, personal identity, higher education. 


\section{Aprendizaje-servicio y educación superior, una apuesta para formar profesionales comprometidos}

El programa para la sostenibilidad de la educación superior elaborado por la Unión Europea en 2013 plantea como una de las funciones de la Universidad la de "agente de cambio, catalizador de la acción social y política, así como centro de aprendizaje" (European Union, 2013, p.4). Esta premisa supone uno de los pilares en los que se apoya la experiencia que aquí presentamos donde resaltamos el papel de la universidad como motor social de cambio y transformación, así como formadora de agentes con conocimientos y sensibilidad social.

Sin embargo, entender la universidad en estos términos requiere una reconceptualización de la cultura docente y necesita de la incorporación de nuevos métodos de enseñanza y aprendizaje que se ajusten de una manera más real a las demandas del entorno. Supone provocar un cambio en los diseños y propuestas metodológicas que favorezcan el desarrollo de aprendizajes que vayan más allá del currículum teórico, fomentando competencias específicas y transversales relacionadas con posicionamientos vitales, conocimiento del otro y herramientas de transformación social.

En esta línea la aproximación el proceso de enseñanza-aprendizaje que se trabaja desde la metodología del aprendizaje-servicio se presenta como una opción altamente válida para cumplir de manera global con los objetivos anteriormente expuestos. Esta metodología se entiende como herramienta de formación que integra el servicio a la comunidad con el estudio académico, con el objeto de enriquecer el aprendizaje desde la práctica (McMillan, 2011; MittonKükner, Nelson y Desrochers, 2010; Kolb, 1984). Esta práctica debe generar responsabilidades cívicas en los estudiantes, así como conectar a la universidad con comunidades con necesidades reales, generando cooperación con la sociedad que dará sentido a la responsabilidad social que tiene la universidad como uno de sus principales cometidos (Blázquez y Martínez-Lozano, 2012; Puig, Gijón, Martín y Rubio, 2011). La sociedad, como contraparte, debe servir de herramienta para permitir y obligar a los estudiantes universitarios a la realización de análisis complejos y reales desde sus aprendizajes, a la vez que implicarlos en procesos de transformación y de participación ciudadana.

El aprendizaje-servicio permite romper con el distanciamiento que se interpone entre los estudios universitarios y los problemas reales y cotidianos de las aulas (Korthagen, 2007; Korthagen y Kessels, 2009), contribuyendo a que los y las estudiantes aprendan y crezcan "moralmente mediante la participación activa en experiencias de servicio organizadas inteligentemente de manera que implican conocimientos" (Naval, 2008, p.64).

En esta línea se encuentran también las recomendaciones que propone la Comisión Europea para la

Martínez-Lozano, V., Rodríguez, R., Marco, M., y Macías, B. (2020). Percepciones del alumnado universitario sobre el aprendizaje-servicio como herramienta para su desarrollo personal y profesional. RIDAS, Revista Iberoamericana de Aprendizaje Servicio, 9, 81-101. DOI10.1344/RIDAS2020.9.5 
modernización de la educación superior, en las que expresa que:

[...] hay que confrontar a los estudiantes con problemas que ellos consideren importantes. Tienen que comprometerse con nuevas preguntas que son más amplias que las asignaturas en sí, que tienen relevancia para sus propias vidas y que provocarán una viva participación que va mucho más allá de la simple presentación a evaluaciones o exámenes (European Union, 2013, p.18).

\section{Aprendizaje-servicio como aprendizaje auténtico}

Trabajar con metodologías docentes de aprendizaje-servicio supone introducir en la docencia nuevas formas de aprendizaje donde la práctica comunitaria se convierte en una piedra angular para la formación del estudiante. Podemos decir así que el aprendizaje adquirido en el ejercicio del aprendizaje-servicio es un aprendizaje situado (Lave y Wenger, 1991; Rogoff, 1990) que tiene relación con el contexto en el que se aprende y que puede llevar a la adquisición de aprendizajes auténticos (Meijers y Wardeker, 2003; Simons, 2000; Van Oers, 2005 y 2007). Hablamos de este tipo de aprendizaje cuando es posible implicar en el proceso formativo no sólo aspectos cognitivos relacionados con contenidos, sino también aspectos emocionales que van más allá e implican a la persona como un todo, afectando a su propia construcción identitaria. Esto se consigue introduciendo al alumnado en problemáticas reales a las que debe enfrentarse, con las que debe comprometerse, y sobre las que debe buscar herramientas que le permitan aprehenderla y entenderla, para así poder transformarla desde su propio ámbito profesional. En este sentido, y siguiendo a Wenger (1998), pensamos que el compromiso con el aprendizaje que se está adquiriendo se convierte en pieza clave para la formación de las y los estudiantes universitarios, fomentando su motivación y su aprender a aprender (Van Dijk y Jochems, 2002), todo ello en el proceso de sentir y tomar conciencia de la utilidad de los mismos.

\section{Antecedentes y contextualización de la investigación}

Los antecedentes de esta experiencia provienen de ámbitos diferentes, aunque complementarios. Por una parte, experiencias nacidas en el seno del enfoque teórico-metodológico de la Psicología Socio-Cultural nos aportan una perspectiva que combina actividades de intervención social con docencia universitaria e investigación. Esta propuesta está sostenida sobre unos pilares teóricos que hacen comprender el desarrollo y el aprendizaje desde una visión social y enlazada con la práctica (Vygotski, 1979). Algunas de estas experiencias son La Quinta Dimensión (Cole, 1996; Cole y the Distributed Literacy Consortium, 2006), La Clase Mágica (Macías y Vásquez, 2014 y 2015; Vásquez, 2003), o La Casa de Shere Rom (Lalueza, Crespo, Pallí y Luque, 1999 y 2001; Lalueza, Crespo, Sánchez, Camps y Cazorla, 2004).

Martínez-Lozano, V., Rodríguez, R., Marco, M., y Macías, B. (2020). Percepciones del alumnado universitario sobre el aprendizaje-servicio como herramienta para su desarrollo personal y profesional. RIDAS, Revista Iberoamericana de Aprendizaje Servicio, 9, 81-101. DOI10.1344/RIDAS2020.9.5 
Por otra parte, encontramos también antecedentes en las experiencias universitarias con metodología aprendizaje-servicio. La existencia de una Red Universitaria de AprendizajeServicio, junto con los encuentros anuales que vienen realizándose desde hace ya casi una década, ha posibilitado el contacto con otras experiencias (Rubio, Prats y Gómez, 2013) que nos han inspirado y animado en el diseño de la práctica que aquí se presenta.

Partiendo de estas aportaciones, en el curso 2012-2013 comenzamos en el Grado de Educación Social una experiencia piloto en la que de manera optativa podía cursarse la asignatura de Procesos de Aprendizaje en Contextos no Formales desde la metodología del aprendizaje-servicio. La evaluación de este curso y el análisis de las notas de campo del alumnado nos reveló importantes aportaciones del aprendizaje-servicio en la formación y aprendizaje del alumnado (Macías, Martínez-Lozano y Mateos, 2014; Macías y Vásquez, 2014) que nos llevó a plantearnos la posibilidad de avanzar en el proyecto con nuevas ambiciones e incorporaciones. Continuamos pues con la experiencia de aprendizaje-servicio implantándola ya como metodología única en un proyecto interdisciplinar entre diferentes asignaturas.

Para el desarrollo y seguimiento del proyecto formamos un grupo de estudios de innovación educativa formado por profesorado de diferentes disciplinas. El proyecto se vio respaldado por su inclusión como acción de innovación docente de la universidad. A continuación describimos los detalles del desarrollo del proyecto docente.

\section{Descripción de la experiencia1}

La experiencia que presentamos se ha llevado a cabo con estudiantes del primer semestre de $1^{\circ}$ del Grado de Educación Social de una universidad pública española, durante los cursos 2014-2015 y 2015-2016. Se trata de un proyecto docente interdisciplinar de aprendizaje-servicio en el que se introduce la acción real, en forma de servicio a la comunidad, como estructura articuladora de dos asignaturas: Bases del Funcionamiento Psicológico Humano y Didáctica en Educación Social. Se contaba además con el apoyo de otra asignatura del semestre, Fundamentos de la Antropología, que introducía en sus prácticas herramientas de análisis de la realidad necesarias para la reflexión y el aprendizaje, como son la etnografía $y$, más concretamente, el cuaderno de campo.

En las dos asignaturas matrices, la parte práctica, que habitualmente se cursa en clases diferentes a las de contenido teórico, se sustituyeron por la asistencia y colaboración con un Centro de Educación Infantil y Primaria constituido como Comunidad de

\footnotetext{
1 Esta experiencia es parte del Proyecto $\mathrm{I}+\mathrm{D}+\mathrm{i}$ Ref. EDU2014-55354-R, 2015/2018 titulado Formación Universitaria: Análisis del proceso de aprendizaje y cambio identitario a través del Aprendizaje-Servicio en comunidades de prácticas en contextos de exclusión, subvencionado por el Programa Estatal de Investigación, Desarrollo e Innovación Orientada a los Retos de la Sociedad, Convocatoria 2014. Así mismo forma parte de los Proyectos de Innovación Docente del Plan de Formación de la Universidad Pablo de Olavide, Acción 2.
} 
Aprendizaje y ubicado en una de las zonas con mayores necesidades de transformación social de la provincia de Sevilla. Su población es

mayoritariamente de etnia gitana.

Los objetivos principales de esta experiencia han sido formulados a varios niveles. Por una parte, con respecto a los aprendizajes del alumnado universitario, tratamos de combinar teoría con práctica, buscando aprendizajes auténticos que permitan al alumnado aplicar conceptos teóricos a sus experiencias y, por tanto, que los estudiantes adquieran no sólo destrezas conceptuales sino conocimientos sobre sí mismos y sobre su papel como futuros profesionales de la educación social. Además, perseguimos el aprendizaje de comportamientos y actitudes orientadas al compromiso ético y a la asunción de responsabilidades sociales como ciudadanos que son. Por otra parte, nos planteamos objetivos de investigación, desde los que pretendemos avanzar en el conocimiento y desarrollo teórico sobre los procesos de aprendizaje que tienen lugar a través del uso de la metodología aprendizaje-servicio. En la línea de estos objetivos, desarrollamos actualmente un $\mathrm{I}+\mathrm{D}+\mathrm{i}$ con la Universidad Autónoma de Barcelona. Un tercer bloque de objetivos se refiere al compromiso social institucional de la universidad para con la sociedad, desde el que entendemos fundamental la colaboración con diferentes entidades sociales y educativas, visibilizando el papel de la universidad como institución a la búsqueda de modelos responsables del sostenimiento de una sociedad igualitaria y justa con todos.
En cuanto a la realización de la experiencia, se ha llevado acabo de la siguiente manera: la docencia presencial de enseñanzas básicas (teóricas) son cursadas en el aula en cada asignatura con la salvedad de la primera semana y la última donde en las que a las clases de ambas asignaturas asiste todo el profesorado implicado (en nuestro caso, tres profesoras) con el objeto de abordar aspectos genéricos e introductorios. En la primera se presenta la asignatura, la metodología a seguir, así como algunas estrategias relevantes para la formación universitaria en general como es el trabajo en grupo, el uso del aula virtual, la elaboración de informes, los trabajos científicos o la búsqueda de bibliografía; la última semana se dedica a la organización de uno de los productos del curso (una jornada de presentación de cortometrajes sobre educación y transformación social en los que han estado trabajando durante el semestre). El resto de las clases teóricas corresponden a cada disciplina y en ellas se discute sobre el temario partiendo de lecturas que los estudiantes deben trabajar, y que deben relacionar con ejemplos de su experiencia en el colegio. Las enseñanzas prácticas dirigidas (clases prácticas) se invierten, en parte, en actividades de servicio a la comunidad. Las horas que corresponden a estas enseñanzas (14 por cada asignatura) se computan en conjunto en las dos asignaturas y se liberan 20 horas para la colaboración del estudiante en el colegio. Estas horas se reparten de la siguiente manera: de la semana tercera a la doceava del semestre los estudiantes realizan el servicio 
comunitario asistiendo dos horas por semana al colegio (un total de 10 semanas). En el colegio, los estudiantes se integran en clases interactivas donde el alumnado está dividido en grupos con actividades distintas. En esas clases hay entre tres y cuatro personas adultas (incluyendo al profesorado) de manera que cada una se encargue de llevar a cabo una actividad. Su objetivo es trabajar el aprendizaje colaborativo entre el alumnado al tiempo que ofrecer una enseñanza individualizada, necesidad que surge por la enorme heterogeneidad que existe en la misma clase, debido a la situación de marginalidad que existe en la zona en la que se ubica el colegio. Las 8 horas restantes se dedican a actividades en el aula, en grupos pequeños, donde se abordan aspectos relacionados directamente con su participación en el colegio y con la evaluación de la experiencia y de sus aprendizajes. Para la primera sesión de trabajo sobre el barrio y el colegio contamos con el apoyo de la Residencia Universitaria Flora Tristán, que se constituye como proyecto social de la universidad ubicada en ese barrio y que cuenta con un conocimiento y una experiencia amplia sobre esta realidad (Blanco, Almirón, Blázquez, Fernández y Maguilla, 2015).

Para la evaluación de los estudiantes planteamos la entrega de varios productos, siempre de manera coordinada entre las asignaturas. Los productos son los diarios de campo, el informe final, la elaboración de un cortometraje y un comentario crítico de varios libros. Además, los estudiantes van realizando informes de seguimiento a través de cuestionarios, debates y microrrelatos. El diario consiste en una nota de campo por cada uno de los días que van al colegio donde registran lo que ha pasado en su jornada de dos horas siguiendo un modelo que les facilitamos y que se divide en tres partes: descripción general del escenario físico y social del aula, descripción detallada de las actividades, interacciones y conductas registradas en la misma, y reflexión personal y teórica sobre la sesión. Hemos de señalar en este punto que una de las piezas fundamentales de esta metodología es la reflexión continua sobre y desde la práctica, mediante el empleo de herramientas teóricas, pues, siguiendo a Dewey (1938), entendemos que para un aprendizaje auténtico y significativo es fundamental la reelaboración del material aprendido, especialmente si está vinculado a la actividad real. En el informe teórico se trabajan conjuntamente conceptos relacionados con ambas asignaturas en especial conexión con las prácticas concretas vividas y reflejadas en las notas de campo.

\section{Evaluación de la experiencia de aprendizaje-servicio}

Tras mostrar el desarrollo de la experiencia, pasamos a mostrar los resultados que estamos obteniendo y que arrojan luz sobre la potencialidad del aprendizaje-servicio como estrategia pedagógica docente. Para ello vamos a guiarnos por los objetivos que planteamos al inicio del texto, y que organizados quedan como sigue:

- Analizar las percepciones de los estudiantes sobre el impacto de

Martínez-Lozano, V., Rodríguez, R., Marco, M., y Macías, B. (2020). Percepciones del alumnado universitario sobre el aprendizaje-servicio como herramienta para su desarrollo personal y profesional. RIDAS, Revista Iberoamericana de Aprendizaje Servicio, 9, 81-101. DOI10.1344/RIDAS2020.9.5 
la experiencia docente en la construcción de su rol profesional, los aprendizajes que realizan a nivel social, sobre la realidad cercana y el otro, y especialmente en su desarrollo como personas.

- Explorar la percepción que tienen sobre la transferibilidad de los aprendizajes adquiridos para su futuro profesional.

- Analizar las valoraciones que realizan sobre la metodología del aprendizaje-servicio.

\subsection{Método}

Para responder a los objetivos planteados se han analizado las narraciones que los estudiantes universitarios han ido realizando a lo largo del semestre. Hemos optado por tanto por una metodología de carácter cualitativo en la que se realizaron análisis del discurso mediante la herramienta EnVivo11.

\subsection{Participantes}

Los estudiantes objeto de estudio fueron todos los que cursaron las asignaturas implicadas en el proyecto de innovación descrito durante los cursos 2014-2015 y 2015-2016.

Participaron un total de 124 estudiantes de la titulación de Educación Social de una universidad pública. El $9,7 \%$ son hombres y el $90,3 \%$ mujeres, oscilando sus edades entre los 18 y los 45 años, siendo la media de 20 años.

\subsection{Instrumentos}

Los datos provienen de narraciones generadas por el alumnado en dos momentos diferentes a lo largo del semestre. El primer momento a mitad de curso donde se plantearon preguntas relacionadas con el desarrollo de mismo. Fueron las siguientes:

1. ¿Qué impacto está teniendo la experiencia de aprendizaje-servicio en ti?

\section{2. ¿Qué aprendizajes estás percibiendo?}

3. ¿Ves la conexión de la experiencia con el contenido curricular de las asignaturas que están implicadas en la experiencia? ¿Te está ayudando la experiencia con otras asignaturas que no están implicadas directamente en ella? Explica tus respuestas

4. ¿Para qué crees que está sirviendo tu colaboración en el colegio? ¿Qué crees que estás aportando al colegio a lo largo del semestre?

5. ¿Qué objetivos te planteas más a largo plazo en cuanto a tu aprendizaje?

6. Describe una experiencia que te haya hecho sentir bien. ¿Por qué crees que te hizo sentir bien?

7. Describe una experiencia que te haya hecho sentir mal. ¿Por qué crees que te hizo sentir mal?

8. ¿Qué tres aspectos destacarías de la experiencia de aprendizaje-servicio?

9. ¿Qué tres aspectos mejorarías de esta experiencia de aprendizajeservicio?

10. ¿Qué no te hemos preguntado y

Martínez-Lozano, V., Rodríguez, R., Marco, M., y Macías, B. (2020). Percepciones del alumnado universitario sobre el aprendizaje-servicio como herramienta para su desarrollo personal y profesional. RIDAS, Revista Iberoamericana de Aprendizaje Servicio, 9, 81-101. DOI10.1344/RIDAS2020.9.5 
crees importante incluir en esta valoración?

El segundo momento de recogida fue a fin de curso, en forma de micro-relatos donde se les pedía que describieran su experiencia durante el semestre. Concretamente se solicitaba una reflexión en base a las siguientes cuestiones:

1. Cómo llegué a la universidad.

2. Qué esperaba encontrarme.

3- Cómo he avanzado en este tiempo.

4. Hacia dónde voy y dónde quiero llegar.

\section{Análisis de los datos}

Los datos han sido analizados desde una aproximación interpretativa basada en el análisis de contenido (Flick, 2004; Krippendorff, 2002), apoyándonos en la Teoría Fundamentada de Strauss y Corbin (1990). Para la codificación, en una primera fase, el profesorado implicado en la experiencia hizo una lectura en paralelo de las narraciones, extrayendo categorías de manera intuitiva. Posteriormente se realizó una puesta en común de las categorías extraídas que fueron agrupadas en torno a los objetivos específicos. Así surgieron las primeras etiquetas:

- Percepciones sobre aspectos relacionados con el aprendizaje de competencias profesionales y sobre cómo van adquiriendo y construyendo su identidad profesional.

- Percepción sobre la realidad social, referido a cómo el alumnado percibe el entorno en el que se está relacionando, sus prejuicios o cambios en sus perspectivas. En este caso es especialmente interesante observar las percepciones sobre las desigualdades sociales, los entornos marginales y sobre la población gitana con la que están trabajando.

- Percepciones sobre aspectos relacionados con su identidad personal, cambios referidos a sí mismos y a la manera de definirse.

- Percepciones sobre el modelo formativo basado en aprendizajeservicio frente a modelos más tradicionales de enseñanza. Valoraciones positivas y negativas.

En una siguiente fase codificamos las narraciones del alumnado en cada una de las categorías anteriores recurriendo al software Nvivo11. En este proceso agrupamos además las respuestas de cada categoría en subcategorías, generando etiquetas que nos permitían describir con más precisión las percepciones de los estudiantes. A continuación presentamos la descripción de las respuestas organizada por categorías y subcategorías.

\section{Resultados}

Los resultados se presentan mostrando frecuencias y porcentajes de respuestas arrojadas en cada categoría. Se analizan estos datos mostrando citas

Martínez-Lozano, V., Rodríguez, R., Marco, M., y Macías, B. (2020). Percepciones del alumnado universitario sobre el aprendizaje-servicio como herramienta para su desarrollo personal y profesional. RIDAS, Revista Iberoamericana de Aprendizaje Servicio, 9, 81-101. DOI10.1344/RIDAS2020.9.5 
textuales que los ilustran, utilizando la inicial A para referirnos a los estudiantes, y añadiendo a continuación un número para identificarlos, ej. $A .1$. Empleamos así mismo el número 14 para referirnos al curso 2014-2015 y el número 15 para el 2015-2016. Mostramos a continuación un resumen general de los resultados, para pasar posteriormente a analizarlos desglosados por categorías y subcategorías.

Tabla 1. Frecuencias y porcentajes de las respuestas del alumnado sobre sus percepciones en diferentes aspectos relacionados con la experiencia de aprendizaje-servicio

\begin{tabular}{ccc}
\hline Categorías & Frecuencias & Porcentajes \\
\hline $\begin{array}{c}\text { Adquisición de } \\
\text { competencias } \\
\text { profesionales y del rol } \\
\text { profesional }\end{array}$ & 210 & $33 \%$ \\
$\begin{array}{c}\text { Aprendizajes sobre la } \\
\text { realidad social }\end{array}$ & 155 & $24,30 \%$ \\
$\begin{array}{c}\text { Aprendizajes y } \\
\text { cambios personales } \\
\quad \text { Total }\end{array}$ & 272 & $42,70 \%$ \\
$\begin{array}{c}\text { Valoraciones positivas } \\
\text { del modelo formativo } \\
\text { basado en }\end{array}$ & $\mathbf{6 3 7}$ & $\mathbf{1 0 0 \%}$ \\
$\begin{array}{c}\text { aprendizaje-servicio } \\
\text { Valoraciones }\end{array}$ & 243 & $68,10 \%$ \\
$\begin{array}{c}\text { negativas del modelo } \\
\text { formativo basado en } \\
\text { aprendizaje-servicio }\end{array}$ & 114 & $31,90 \%$ \\
$\quad$\begin{tabular}{l} 
Total \\
\hline
\end{tabular} & $\mathbf{3 5 7}$ & $\mathbf{1 0 0 \%}$ \\
\hline
\end{tabular}

Fuente: elaboración propia

Observando los totales contabilizados se puede apreciar que aparecen 637 afirmaciones relacionadas con adquisiciones de competencias profesionales, aprendizajes sobre la realidad o percepción de cambios personales. Aparecen así mismo 357 valoraciones sobre la metodología empleada, de las cuales la mayor parte corresponde a aspectos positivos sobre esta. Pasamos a continuación a describir en detalle cada una de las categorías, mostrando ejemplos que ilustren cada una de ellas.

\subsection{Percepciones sobre la adquisición} de competencias profesionales y sobre construcción del rol profesional

En la tabla 2 se muestran las frecuencias y los porcentajes de los resultados del aprendizaje en relación con la adquisición de competencias elementales para el desempeño de su profesión, así como en la identificación de aspectos relevantes para la construcción de su identidad profesional.

Tabla 2. Frecuencias y porcentajes de resultados sobre percepciones sobre su identidad profesional declarados por los estudiantes

\begin{tabular}{ccc}
\hline Subcategorías & Frecuencias & Porcentajes \\
\hline $\begin{array}{c}\text { Adquisición de } \\
\text { metodologías, recursos } \\
\text { y estrategias }\end{array}$ & 88 & $41.9 \%$ \\
$\begin{array}{c}\text { Capacidad para } \\
\text { trabajar en equipo }\end{array}$ & 41 & $19.5 \%$ \\
$\begin{array}{c}\text { Descubrimiento del rol } \\
\text { de mediación en } \\
\text { contextos educativos. }\end{array}$ & 21 & $10 \%$ \\
$\begin{array}{c}\text { Conocimiento de } \\
\text { nuevos ámbitos y } \\
\text { nichos profesionales }\end{array}$ & 15 & $7.1 \%$ \\
$\begin{array}{c}\text { Compromiso con la } \\
\text { justicia social }\end{array}$ & 45 & $\mathbf{2 1 . 4 \%}$ \\
\hline Total & $\mathbf{2 1 0}$ & $\mathbf{1 0 0} \%$ \\
\hline
\end{tabular}

Fuente: elaboración propia

Las percepciones sobre los aprendizajes en relación con la dimensión profesional 
aparecen principalmente alrededor de adquisiciones de estrategias

relacionadas con la profesión (41.9\%) y en concreto con el trabajo en grupo $(19.5 \%)$ que hemos destacado por ser la estrategia más destacada. Entre ambos aspectos suman más del $60 \%$ por lo que vemos que dentro de esta categoría es el aspecto que más se destaca. Lo siguiente que aparecen son narraciones en las que aluden a su compromiso con la justicia y la transformación social (21.4\%). Podemos observar además como la experiencia de aprendizaje-servicio también aporta una mejor comprensión del rol mediador del aprendizaje de los profesionales de la educación (10\%), permitiéndoles además el descubrimiento de ámbitos y nichos profesionales para la Educación social (7.1\%). A continuación aportamos algunos ejemplos de los discursos producidos por los estudiantes en este sentido:

- A29.14. "Me he dado cuenta de lo importante que es trabajar en grupo. Es algo que ha estado presente en estas asignaturas, en las profesoras, y que hemos aprendido también en el colegio trabajando con el maestro y otros voluntarios en las comunidades de aprendizaje. Gracias al aprendizajeservicio estoy aprendiendo a trabajar en grupo, a cooperar, a no disgustarme si alguien hace algo distinto a lo que yo pienso que tiene que ser, a coordinarme en el trabajo...".

- A22.14. "He tenido la oportunidad de comprobar las estrategias que funcionan y las que no... No solo haciéndolo, sino observando al maestro. La idea de unir la teoría a la práctica mediante un servicio me entusiasmó porque me hizo darme cuenta de que es así como yo tengo que trabajar desde la educación social".

- A18.14. "La experiencia en el colegio con lo del aprendizajeservicio nos ha completado a todos y nos ha dado una visión amplia de la función imprescindible que tenemos hoy y que tendremos en el futuro como educadores sociales en la sociedad. Somos mediadores de aprendizajes. Ahora entiendo mejor lo que he leído de Freire, eso de la concienciación y la liberación... Antes no me lo hubiera creído, lo vería muy utópico pero he visto que si se puede y es lo que tenemos que hacer los educadores sociales".

- A82.15. "Aún es pronto para saber en qué ámbito me gustaría trabajar pero he descubierto el mundo de la infancia... Ahora no estoy cerrada a trabajar en centros de menores, colegios, y otros lugares donde haya niños o menores".

- A59.14. "No conocía el trabajo con comunidades de aprendizaje... Pensaba que la educación social se dedicaba más a ámbitos no formales. Me ha abierto un mundo de posibilidades nuevas a nivel profesional. Quiero trabajar en desarrollo comunitario pero desde dentro de la escuela...".

- A94.15. "No sabía que existían tantas diferencias y desigualdades... lo que he visto gracias al aprendizaje-servicio no lo conocía. Y ahora sé que mi profesión es para intentar cambiar justo eso. Me siento motivada y comprometida". 
Con estos enunciados mostramos cómo el alumnado implicado en el proyecto adquiere, desde los primeros momentos de su participación, tanto destrezas relacionadas con su futura profesión (trabajo en grupo, metodologías activas, capacidad de mediación), como un acercamiento a ámbitos de trabajo de la misma (menores, desarrollo comunitario), y al tiempo desarrolla actitudes necesarias para su labor profesional, como es en este caso la conciencia de la desigualdad social y la voluntad de minimizar sus efectos, así como un compromiso con la transformación y la justicia social.

\subsection{Percepciones sobre la realidad social}

En esta categoría el alumnado hace referencia a sus aprendizajes y cambios de visión con respecto a la comunidad gitana y al entorno del Polígono Sur, ya que esta zona está enormemente estigmatizada y es considerada como muy peligrosa por gran parte de la población. Son múltiples las expresiones en este sentido (155 menciones de 994).

Tabla 3. Frecuencias y porcentajes de resultados sobre percepciones sobre la realidad social.

\begin{tabular}{ccc}
\hline Subcategorías & Frecuencias & Porcentajes \\
\hline $\begin{array}{c}\text { Estereotipos sobre } \\
\text { la etnia gitana }\end{array}$ & 86 & $55.4 \%$ \\
\hline $\begin{array}{c}\text { Estereotipos sobre } \\
\text { el barrio }\end{array}$ & 69 & $44.5 \%$ \\
\hline Total & $\mathbf{1 5 5}$ & $\mathbf{1 0 0 \%}$ \\
\hline
\end{tabular}

Fuente: elaboración propia
Algunos fragmentos interesantes y elocuentes para ilustrar esta categoría son los que presentamos a continuación:

- A12.14. "He conocido el barrio y he cambiado mucho de los estereotipos negativos que tenía, no todo es malo en el Polígono Sur... hay muchos padres y madres interesados en la educación de sus hijos y eso te llena de alegría y esperanza".

- A84.15. "He aprendido a diferenciar entre las personas de etnia gitana $y$ la marginación... Son dos cosas diferentes. Muchas veces los confundimos pero no es por ser gitanos, es por estar en un barrio de mucha exclusión".

- A59.14. "Personalmente se me han caído muchas ideas preconcebidas que tenía sobre la comunidad gitana, muchos estereotipos... Al principio me dio mucho miedo pensar que tenía que ir allí... Ahora veo que son personas como nosotros y sé distinguir entre la etnia gitana y la exclusión social que son dos cosas distintas..."

Tal y como se puede observar en los tres enunciados presentados, la experiencia de aprendizaje-servicio representa un impacto importante en el cuestionamiento de las ideas previas y los estereotipos sobre el barrio en el que realizan la actividad, sobre la comunidad gitana $y$, en general, sobre los contextos relacionados con la marginación y la exclusión social. Las consideraciones que realizan sobre la separación de cultura gitana y cultura de la marginación constituyen un aspecto enormemente importante

Martínez-Lozano, V., Rodríguez, R., Marco, M., y Macías, B. (2020). Percepciones del alumnado universitario sobre el aprendizaje-servicio como herramienta para su desarrollo personal y profesional. RIDAS, Revista Iberoamericana de Aprendizaje Servicio, 9, 81-101. DOI10.1344/RIDAS2020.9.5 
desde nuestro punto de vista, ya que es la base para entender la sociedad y la desigualdad. Consideramos que estos cambios sobre el conocimiento del otro son imprescindibles para que estos y estas estudiantes se conviertan en futuros profesionales comprometidos socialmente.

\subsection{Percepciones sobre cambios personales}

En la tabla 4 se muestran los datos relacionados con narraciones que hacen referencia a ellos mismas, ofreciendo muestras de cómo la experiencia les está afectando emocional y personalmente y por tanto cómo estos aprendizajes inciden también en sus procesos de construcción personal e identitaria.

Tabla 4. Frecuencias y porcentajes de resultados de aprendizajes personales.

\begin{tabular}{ccc}
\hline Subcategorías & Frecuencias & Porcentajes \\
\hline Empatía & 47 & $17.3 \%$ \\
\hline Paciencia & 80 & $29.4 \%$ \\
\hline $\begin{array}{c}\text { Empoderamiento/ } \\
\text { autoconfianza }\end{array}$ & 76 & $27.9 \%$ \\
\hline $\begin{array}{c}\text { Flexibilidad } \\
\text { Confirmación en } \\
\text { la elección } \\
\text { vocacional }\end{array}$ & 22 & $8.1 \%$ \\
\hline Total & $\mathbf{1 5 5}$ & $\mathbf{1 0 0} \%$ \\
\hline
\end{tabular}

Fuente: elaboración propia

Tal como se muestra en la tabla, los resultados relacionados con aspectos personales revelan que mayoritariamente el estudiantado hace referencia al desarrollo de la paciencia (29.4\%) como aspecto más reseñable, seguido por el empoderamiento y la autoconfianza que muestran tras unos meses de colaboración en el colegio (27.9\%). Destacan igualmente la empatía $(17.3 \%)$ como un aspecto que consideran de relevancia en sus aprendizajes y hacen referencia también a la flexibilidad que están observando en el desarrollo de sus acciones $(8.1 \%)$. Finalmente hemos encontrado un aspecto relacionado con su formación identitaria como es la confirmación de su vocación profesional $(17.3 \%)$, aspecto enormemente relevante en los procesos motivacionales de aprendizaje. Algunos ejemplos de los discursos producidos por los estudiantes en este sentido son:

- A12.14. "Me he tenido que situar en el lugar del otro para evitar hacer juicios... Me he tenido que poner muchas veces en la situación de estos niños...".

- A92.15. "Lo que más me ha ayudado en el contacto con estos niños es a desarrollar mi paciencia... Como educadora no puedes perder los nervios y ellos intentan ponerte a prueba. Los primeros días en el colegio me di cuenta de que no tenía paciencia y que la necesitaba. Ahora me doy cuenta de que he ido creciendo en ella y me siento muy satisfecha..."

- A32.14. "Creo que en estos tres primeros meses ha cambiado mi vida totalmente: mayor autonomía, más confianza en mí misma, libertad total para ir creando mi propio camino, nuevos objetivos que cumplir y metas que alcanzar. Me considero una persona muy rígida,

Martínez-Lozano, V., Rodríguez, R., Marco, M., y Macías, B. (2020). Percepciones del alumnado universitario sobre el aprendizaje-servicio como herramienta para su desarrollo personal y profesional. RIDAS, Revista Iberoamericana de Aprendizaje Servicio, 9, 81-101. DOI10.1344/RIDAS2020.9.5 
me gusta tenerlo todo claro y controlar pero ahora veo que esto no es así y que tengo que adecuarme a cada circunstancia y trabajar con lo que me encuentro".

- A12.14. "Ahora sé que esto es lo que quiero hacer y lo que me gusta. Al principio no estaba segura. Esta experiencia me ha transmitido la esencia que tiene para mí la Educación Social: luchar juntos por una vida mejor".

En estos fragmentos los estudiantes muestran sus percepciones con relación a sí mismos, mostrando el peso que la experiencia de aprendizaje-servicio está teniendo en su autoconcepto y en propia identidad personal. Encontramos alguna afirmación que expone cómo está cambiando su vida su vida por la forma en la que ahora se autopercibe, como parte de una comunidad diferente en la que debe asumir roles y posicionamientos diferentes (A.32.14).

\subsection{Valoraciones sobre el modelo} formativo docente basado en aprendizaje-servicio

Para esta categoría se hizo un recuento de valoraciones positivas y negativas, agrupando qué aspectos se señalan en cada una de ellas. Las frecuencias positivas se refieren a aprendizajes que explícitamente señalan como derivadas de la metodología empleada. Las negativas indican aspectos problemáticos o con los que se han sentido a disgusto. Las tablas 5 y 6 reflejan los resultados de cada una de las subcategorías que hemos utilizado para analizar los diferentes aspectos valorados.
Tabla 5. Frecuencias y porcentajes de los fragmentos textuales declarados por el alumnado sobre aspectos positivos relacionados con la experiencia con aprendizaje-servicio.

\begin{tabular}{ccc}
\hline Subcategorías & Frecuencia & Porcentajes \\
\hline $\begin{array}{c}\text { Enseñanza más } \\
\text { activa (aprender } \\
\text { haciendo) }\end{array}$ & 59 & $24,30 \%$ \\
\hline $\begin{array}{c}\text { Conexión teórico- } \\
\text { práctica }\end{array}$ & 63 & $25,90 \%$ \\
$\begin{array}{c}\text { Satisfacción } \\
\text { general con la } \\
\text { experiencia }\end{array}$ & 48 & $19,80 \%$ \\
$\begin{array}{c}\text { Pertinencia de la } \\
\text { evaluación } \\
\text { continua }\end{array}$ & 37 & $15,20 \%$ \\
$\begin{array}{c}\text { Permite conocer la } \\
\text { profesión desde } \\
\text { dentro }\end{array}$ & 36 & $14,80 \%$ \\
\hline Total & $\mathbf{2 4 3}$ & $\mathbf{1 0 0} \%$ \\
\hline
\end{tabular}

Fuente: elaboración propia

Tabla 6. Frecuencias y porcentajes de los fragmentos textuales declarados por el alumnado sobre aspectos negativos relacionados con la experiencia con aprendizaje-servicio.

\begin{tabular}{ccc}
\hline Subcategorías & Frecuencia & Porcentajes \\
\hline $\begin{array}{c}\text { Genera } \\
\text { inseguridad al } \\
\text { inicio }\end{array}$ & 29 & $25,40 \%$ \\
$\begin{array}{c}\text { Diferencias } \\
\text { metodológicas } \\
\text { entre asignaturas } \\
\text { Gran carga de } \\
\text { trabajo }\end{array}$ & 33 & $28,90 \%$ \\
$\begin{array}{c}\text { Dudas acerca de } \\
\text { los aprendizajes } \\
\text { académicos }\end{array}$ & 28 & $21,10 \%$ \\
\hline Total & $\mathbf{1 1 4}$ & $\mathbf{1 0 0} \%$ \\
\hline
\end{tabular}

Fuente: elaboración propia

De un total de 357 unidades de texto en esta categoría, 243 son narraciones

Martínez-Lozano, V., Rodríguez, R., Marco, M., y Macías, B. (2020). Percepciones del alumnado universitario sobre el aprendizaje-servicio como herramienta para su desarrollo personal y profesional. RIDAS, Revista Iberoamericana de Aprendizaje Servicio, 9, 81-101. DOI10.1344/RIDAS2020.9.5 
que destacan aspectos positivos y 114 negativos. Un análisis pormenorizado de estas afirmaciones muestra que el alumnado valora especialmente la posibilidad de aprender desde la práctica $(24,3 \%)$, así como la conexión que se establece entre la teoría y la práctica $(25,9 \%)$ que les ayuda a entender mejor los conceptos. Uniendo estas dos primeras subcategorías encontramos que como método de aprendizaje de conocimientos teóricos esta metodología es enormemente valorada, y destacada como la mayor ventaja de esta metodología $(50,2 \%)$. Es importante destacar también la satisfacción general que despierta la experiencia, de manera que, tal y como afirman algunos/as estudiantes, compensa la carga de trabajo $(19,8 \%)$. La preferencia por la evaluación continua y no mediante pruebas objetivas es también valorado como positivo $(15,2 \%)$, así como la posibilidad que les ofrece esta experiencia de conocer la profesión desde dentro $(14,8 \%)$.

En la parte negativa el estudiantado destaca su preocupación por no poder conectar esta experiencia con todas las asignaturas del semestre $(28,9 \%)$, lo que entienden como deseable y muestra un mayor deseo de interdisciplinariedad en sus estudios. Hacen referencia también al miedo inicial ante una población y un barrio desconocidos y con marcadores muy negativos, cargados de prejuicios. Le sigue su preocupación por el aprendizaje conceptual que están realizando $(24,6 \%)$, lo cual debería preocuparnos, aunque anteriormente se han manifestado en sentido contrario.
Nuestras intuiciones nos indican que la falta de exámenes puede estar generando en ellos y ellas la sensación de poco aprendizaje, aunque en los textos que generan hemos apreciado un manejo muy diestro de los conceptos trabajados en clase. Este aspecto merecería la pena ser analizado en posteriores estudios. Por último señalan que esta metodología les requiere gran carga de trabajo ya que deben leer y producir textos semanalmente, además de asistir al colegio.

A continuación se presentan algunos fragmentos ilustrativos de esta categoría:

- A63.14. "Lo que he aprendido con el aprendizaje-servicio no lo podría haber aprendido en la universidad solamente. He aprendido haciendo y he puesto en práctica habilidades que solo las desarrollas en el contacto real con la gente. Especialmente en una profesión como la nuestra. Creo que de no haber llevado las clases con aprendizaje-servicio me hubiera encasillado y, tal vez, incluso lo hubiera dejado... pero esto me ha cautivado y quiero seguir".

- A101.15. "Pensaba que todo iba a ser mucho más teórico, sobre todo en los primeros cursos pero ahora veo que gracias al aprendizajeservicio no es así y que no tengo que esperar a que lleguen las prácticas. No veo otro modo mejor para aprender la profesión que acercándonos a ella y reflexionando en las clases".

- A74. 15. "Pensaba que los profesores iban a ser distantes y que nosotros íbamos a ser unos meros receptores, pero la verdad es que ha

Martínez-Lozano, V., Rodríguez, R., Marco, M., y Macías, B. (2020). Percepciones del alumnado universitario sobre el aprendizaje-servicio como herramienta para su desarrollo personal y profesional. RIDAS, Revista Iberoamericana de Aprendizaje Servicio, 9, 81-101. DOI10.1344/RIDAS2020.9.5 
sido lo contrario, tanto profesores como alumnos nos hemos implicado y juntos hemos hecho algo útil... Ha sido una experiencia única... me ha hecho crecer como persona".

\section{Conclusiones}

Este trabajo muestra la experiencia con metodología de aprendizaje-servicio que venimos realizando desde hace dos años en la universidad. Desde la perspectiva del alumnado sobre los principales aprendizajes que señalan haber adquirido, así como sobre sus valoraciones sobre esta forma de trabajo en la formación universitaria. Los resultados muestran la potencialidad que en diferentes aspectos tienen el aprendizaje-servicio: permite conocer de primera mano un campo profesional en el que trabajar así como adquirir competencias adecuadas para ello; fomenta la motivación y la iniciativa para la acción social, confrontando con realidades y grupos socio-culturales diferentes sobre los que, como el estudiantado indica, tenían escaso conocimiento e incluso podían albergar prejuicios negativos; y posibilita reconocer y activar destrezas personales útiles de las que hasta entonces no habían sido conscientes o no habían prestado atención.

Destacamos que los aprendizajes alcanzados por el estudiantado no se circunscriben a contenidos disciplinares de carácter conceptual. Por el contrario, dado que el aprendizaje-servicio conjuga la dimensión académica con un planteamiento experiencial -en el que el plano emocional del aprendizaje tiene un valor esencial-, los aprendizajes conceptuales se entretejen con otros procedimentales y con cambios actitudinales y valores que les dan sentido personal. En el caso que analizamos, junto al cuestionamiento de las propias creencias y actitudes previas y al compromiso con la búsqueda de una mayor justicia social, el alumnado refiere como resultado directo de la experiencia de aprendizaje-servicio la adquisición o mejora de, entre otras, las siguientes competencias: habilidades de relación interpersonal, habilidad para adaptarse y trabajar en un contexto intercultural complejo, capacidad para gestionar la diversidad cultural y la mejora de competencias del trabajo en equipo. Estas habilidades son de indudable valor en la formación integral de personas $y$, especialmente, en la de profesionales de la intervención social.

En definitiva, estos resultados muestran la gran utilidad formativa de la metodología aprendizaje-servicio, especialmente con relación al desarrollo personal y a los aprendizajes profesionales, destacando cuatro aspectos fundamentales: en primer lugar, facilita una percepción más realista de la profesión, tanto por la complejidad del campo de trabajo como por las destrezas implicadas en el desempeño; en segundo lugar, contribuye a que el alumnado reflexione sobre su implicación en las labores profesionales y adquieran confianza en sí mismos en el ejercicio de esa práctica profesional; en tercer lugar, ayuda a participar y a sentirse agentes en una comunidad de práctica profesional, lo cual despierta una mayor motivación en su formación como educadores sociales; en cuarto y último lugar, pero

Martínez-Lozano, V., Rodríguez, R., Marco, M., y Macías, B. (2020). Percepciones del alumnado universitario sobre el aprendizaje-servicio como herramienta para su desarrollo personal y profesional. RIDAS, Revista Iberoamericana de Aprendizaje Servicio, 9, 81-101. DOI10.1344/RIDAS2020.9.5 
no menos importante, favorece el acercamiento a la diversidad social, el conocimiento de las desigualdades y la reivindicación de la equidad y la justicia social. Estos resultados se ven reforzados por la positiva valoración que el alumnado realiza de la metodología seguida, especialmente por la conexión teoría-práctica que facilita, lo que, a su juicio, compensa la mayor carga de trabajo y les hace desear la vinculación de la experiencia a otras materias.

Estos resultados concuerdan en gran parte con los obtenidos por Folgueiras, Luna y Puig (2013), donde analizan la satisfacción del estudiantado tras pasar por una experiencia aprendizajeservicio, concluyendo que la dimensión más destacada fue la valoración atribuida a la experiencia, es decir, sus percepciones sobre la adquisición de aprendizajes conceptuales, personales y ciudadanos.

Por todo ello consideramos que el aprendizaje-servicio es una herramienta docente que puede conducir a las y los estudiantes a un aprendizaje auténtico (Meijers y Wardeker, 2003; Simons, 2000; Van Oers, 2005 y 2007), que no sólo suponga cambios a nivel cognitivo, sino que lleve a aprendizajes que van más allá del currículum e incluyan cambios en los posicionamientos personales. Es decir, lo que aprenden, lo aprenden desde la práctica, conectando con ella y replanteándose a sí mismos como agentes, lo que implica un compromiso personal y profesional con la realidad social y con las personas con las que se trabaja, aspecto que Wenger (1998) considera esencial en todo aprendizaje.
Finalmente, y no menos importante, este estudio muestra el aprendizajeservicio como herramienta que permite que las universidades cumplan con su responsabilidad social como institución transformadora de la sociedad. $Y$ esto en un doble sentido: de un lado, facilitando la original misión de las instituciones universitarias como ámbito para la formación integral de futuros profesionales como personas comprometidas y responsables que articularán la sociedad en un futuro cercano; y de otro, yendo más allá y promoviendo un espacio base para la articulación y la colaboración con instituciones públicas y entidades sociales que presentan demandas reales en una sociedad compleja y en crisis como en la que ahora nos movemos.

\section{Perspectivas de futuro}

Dada la potencialidad del aprendizajeservicio para propiciar aprendizajes auténticos parecen obvios los beneficios que traería su transferencia a otros cursos superiores, y a otras disciplinas. Encontramos esta metodología especialmente interesante para grados en el marco de las Ciencias Sociales y Humanas, en los que la implicación de los estudiantes en las problemáticas sociales ha de constituir gran parte de su repertorio formativo. Pero no solo en Ciencias Sociales, las Ilamadas carreras experimentales y técnicas se nos antojan un campo en el que el aprendizaje de servicio podría cobrar especial sentido.

En nuestra experiencia hemos planteado el servicio comunitario y el aprendizaje-servicio en el marco de la

Martínez-Lozano, V., Rodríguez, R., Marco, M., y Macías, B. (2020). Percepciones del alumnado universitario sobre el aprendizaje-servicio como herramienta para su desarrollo personal y profesional. RIDAS, Revista Iberoamericana de Aprendizaje Servicio, 9, 81-101. DOI10.1344/RIDAS2020.9.5 
colaboración entre diferentes asignaturas del mismo curso, implicando altas dosis de cooperación docente y planificación conjunta. Para el futuro, consideramos que la colaboración y coordinación docente constituyen una piedra angular de este tipo de proyectos. Idealmente, un escenario a alcanzar sería el trabajo en torno al aprendizaje-servicio a lo largo de un curso completo, en el que todas las asignaturas del mismo estuvieran de alguna manera implicadas en el proyecto. Para esto sería necesaria la implicación de la institución universitaria, generando un eje prioritario del programa formativo en las memorias verifica de los grados y reorganizando, si fuera necesario, la planificación docente para adaptarla a esta metodología. A la vez se deben establecer relaciones intensas de trabajo con instituciones y entidades educativas y de atención social que permitan, de un lado, configurar ese escenario idóneo para el aprendizaje y, de otro, que el servicio comunitario sobre el que se establece responda a necesidades reales de la población con la que se trabaja.

Entendemos asimismo que conocer el know-how del trabajo, a través de la triangulación con las instituciones públicas y las entidades colaboradoras, es claramente necesario para valorar las potencialidades y las limitaciones de este tipo de experiencias, además de hacerlas claramente exportables a otras titulaciones y niveles de enseñanza, universitarias (posgrados), o no (secundaria).

En consecuencia, se espera que esta experiencia sirva como palanca para impulsar nuevos desarrollos en el aprendizaje-servicio y futuras investigaciones, no sólo para promover aprendizajes en el alumnado universitario y evaluar su impacto, sino también para valorar la incidencia de este modo de trabajar en el desarrollo de alineamientos entre universidades y comunidad, así como de redes interuniversitarias.

\section{Referencias bibliográficas}

Blanco, J., Almirón, M. A., Blázquez, A., Fernández, A., y Maguilla, M. (febrero de 2015). La Residencia Universitaria Flora Tristán un experiencia de intervención/acción en el territorio desde la universidad. En M. León (Presidencia), Desigualdad y democracia: políticas públicas e innovación social. Ponencia llevada a cabo en el V Congreso de la Red Española de Política Social (REPS), Barcelona, España. Recuperado de https://cendocps.carm.es/documentaci on/2016_Actas_finales_Red_Espa\%c3 \%b1ola_Politica_Social2015.pdf

Blázquez, A. y Martínez-Lozano, V. (2012). La residencia universitaria Flora Tristán: Un ejemplo de formación humana y de compromiso con la sociedad. Revista de Educación, 358, 618-630.

Cole, M. (1996). Cultural Psychology. A Once and Future Discipline. Cambridge, United States of America: Harvard University Press.

Cole, M. y The Distributed Literacy Consortium. (2006). The Fifth Dimension: An after-school program built on diversity. New York, United 
States of America: Russell Sage Foundation.

Dewey, J. (1938). Education and experience. New York, United States of America: Simon \& Schuster.

European Union. (2013). Improving the quality of teaching and learning in Europe's higher education institutions. Report of the European Commission. Luxembourg: Publications Office of the European Union.

Flick, U. (2004). Introducción a la investigación cualitativa. Madrid, España: Morata.

Folgueiras, P., Luna, E., y Puig, G. (2013). Aprendizaje y servicio: estudio del grado de satisfacción de estudiantes universitarios. Revista de Educación, 362, 159-185.

Kolb, D. (1984). Experiential learning: Experience as the source of learning and development. New Jersey, United States of America: Prentice-Hall.

Korthagen, F. (2007). The gap between research and practice revisited.

Educational Research and Evaluation, 13(3), 303-310.

Korthagen, F. y Kessels, J. (2009). Linking theory and practice: Changing the pedagogy of teacher education. Educational Researcher, 28(4), 4-17.

Krippendorff, K. (2002). Metodología de análisis de contenido. Teoría y práctica. Barcelona, España: Paidós.

Lalueza, J.L., Crespo, I., Pallí, C. y Luque, M.J. (1999). Intervención educativa, comunidad y cultura gitana. Una experiencia con nuevas tecnologías: la Casa de Shere Rom. En M.A. Essombra (Ed.), Construir la escuela intercultural. Reflexiones y propuestas para trabajar la diversidad étnica y cultural (pp. 185-194). Barcelona, España: Graó.

Lalueza, J.L., Crespo, I., Pallí, C. y Luque, M.J. (2001). Socialización y cambio cultural en una comunidad étnica minoritaria. El nicho evolutivo gitano. Cultura y Educación, 13(1), 115-130.

Lalueza, J. L., Crespo, I., Sánchez, S., Camps, S. y Cazorla, A. (2004). Intervención psicopedagógica en comunidades minoritarias. En C. Monero (Ed.) La práctica psicopedagógica en educación no formal. Barcelona, España: EDIUOC.

Lave, J. y Wenger, E. (1991). Situated Learning: Legitimate Peripheral Participation. Cambridge, UK: Cambridge University Press.

Macías, B., Martínez-Lozano, V. y Mateos, C. (2014). Usos de la etnografía en un proyecto de Aprendizaje-Servicio en el contexto universitario. En P. Mata, B. Balleteres e I. Gil-Jaurena (Eds.), Aprendizaje de la ciudadanía y la participación (pp. 115121).

Macías, B., Martínez-Lozano, V., y Vásquez, O. A. (2015). "Real Learning" in Service Learning: Lessons from La Clase Mágica in the US and Spain. International Journal for Research on Extended Education, 2(2), 63-68. 
Macías, B. y Vásquez, O.A. (2014). La Clase Mágica goes international: adapting to new sociocultural contexts. En B. Bustos, O. Vasquez \& E. Clark (Eds.). Generating transworld pedagogy (pp.193-208). Maryland, United States of America: Lexington Books.

Macías, B. y Vásquez, O.A. (2015). Identity construction in narratives of migration. En T. Hansen \& K. Jensen (Eds). Self in Culture in Mind. Connectional and applied perspectives (pp.177-200). Aalborg, Denmark: Aalborg University Press.

McMillan, J. (2011). What happens when the university meets the community? Service learning, boundary work and boundary workers. Teaching in Higher Education, 16(5), 553-564.

Meijers, F. y Wardekker, W. (2003). Cateer learning in a changing world: The role of emotions. International Journal for the Advancement of Counseling, 24, 149-167.

Mitton-Kükner, J., Nelson, C. y Desrochers, C. (2010). Narrative inquiry in service-learning contexts: Possibilities for learning about diversity in teacher education. Teaching and Teacher Education, 26, 1162-1169.

Naval, C. (2008). Universidad y conciencia cívica. Algunas experiencias fructíferas: service learning y campus compact. En M. Martínez (Ed.). Aprendizaje servicio y responsabilidad social de las Universidades (pp. 57-80). Madrid, España: Octaedro.

Rogoff, B. (1990). Apprenticeship in thinking: Cognitive development in social context. Oxford, UK: Oxford University Press.

Puig, J., Gijón, M., Martín, X. y Rubio, L. (2011). Aprendizaje-servicio y educación para la ciudadanía. Revista de Educación, número extraordinario, 45-67. Recuperado de

http://www.revistaeducacion.educacion .es/re2011/re2011_03.pdf

Rubio, L., Prats, E. y Gómez, L. (Coord.). (2013). Universidad y Sociedad. Experiencias de Aprendizaje Servicio en la Universidad. Barcelona: Universitat de Barcelona (Institut de Ciències de I'Educació). Recuperado de https://www.uv.es/aps/doc/Biblioteca\% 20Blog/Univerdidad\%20y\%20sociedad. pdf

Simons, J. (2000). Towards a constructivist theory of self-directed learning. En G. Straka (Ed.), Selflearning (pp. 155-169). Münster, Germany: Waxmann.

Strauss, A. L. y Corbin, J. (1990). Basics of qualitative research. Newbury Park, United States of America: Sage publications.

Van Dijk, L. A. y Jochems, W.M.G. (2002). Changing a traditional lecturing approach into an interactive approach: Effects of interrupting the monologue in lectures. International Journal of Engineering Education, 18(3), 275-284. Recuperado de https://www.ijee.ie/articles/Vol183/IJEE1300.pdf

Van Oers, B. (2005). The potentials of imagination. Inquiry, 24(4), 5-18. 
Van Oers, B. (2007). Helping young

children to become literate: The relevance of narrative competence for developmental education. European Early Childhood Education Research Journal, 15(3), 299-312.

Vásquez, O. (2003). La Clase Mágica: Imagining optimal possibilities in a bilingual community of learners. New Jersey: Laurence Erlbaum.

Vygotski, L.S. (1979). El desarrollo de los procesos psicológicos superiores. Barcelona, España: Crítica.

Wenger, E. (1998). Communities of practice: learning, meaning, and identity. Cambridge, UK: Cambridge University Press. 